\title{
Extension of the optimized RHNC equation to multicomponent liquids
}

Cite as: J. Chem. Phys. 87, 2249 (1987); https://doi.org/10.1063/1.453153

Submitted: 12 March 1987. Accepted: 06 May 1987. Published Online: 04 June 1998

E. Enciso, F. Lado, M. Lombardero, J. L. F. Abascal, and S. Lago

\section{ARTICLES YOU MAY BE INTERESTED IN}

Hypernetted chain solutions for the classical one-component plasma up to $\Gamma=7000$ The Journal of Chemical Physics 61, 2680 (1974); https://doi.org/10.1063/1.1682399

A crossover integral equation for the structure of simple liquids

The Journal of Chemical Physics 80, 4441 (1984); https://doi.org/10.1063/1.447225

Free energy model for inhomogeneous fluid mixtures: Yukawa-charged hard spheres, general interactions, and plasmas

The Journal of Chemical Physics 98, 8126 (1993); https://doi.org/10.1063/1.464569

\section{Lock-in Amplifiers up to $600 \mathrm{MHz}$}
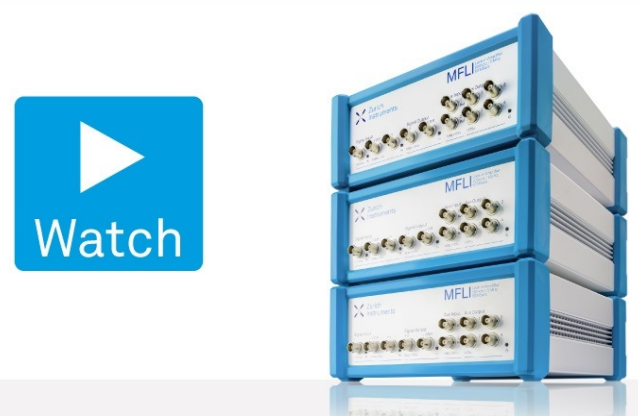


\title{
Extension of the optimized RHNC equation to multicomponent liquids
}

\author{
E. Enciso \\ Departamento de Quimica Fisica, Facultad de Química, Universidad Complutense, 28040 Madrid, Spain
}

F. Lado

Department of Physics, North Carolina State University, Raleigh, North Carolina 27695-8202

M. Lombardero

Instituto de Química Fisica Rocasolano, C. S. I. C., Serrano 119, and Departamento de Química Fisica, Facultad de Química, Universidad Complutense, 28040 Madrid, Spain

J. L. F. Abascal and S. Lago

Departamento de Química Fisica, Facultad de Química, Universidad Complutense, 28040 Madrid, Spain

(Received 12 March 1987; accepted 6 May 1987)

\begin{abstract}
We have extended the optimized reference-hypernetted chain formalism to multicomponent liquids. The reference system is constructed from a mixed hard spheres fluid with additive diameters whose structural and thermodynamic properties have been conveniently parametrized. The theory is applied to binary liquid mixtures interacting through a repulsive Lennard-Jones potential as well as the complete Lennard-Jones potential; calculated results are in excellent agreement with those of numerical simulations.
\end{abstract}

\section{INTRODUCTION}

Knowledge of the pair distribution function (PDF) $g(r)$ is the key theoretical problem in acquiring information about the thermodynamic and structural properties of simple liquids in equilibrium whose molecular interactions can be described by an effective, spherically symmetric pair potential $\phi(r))^{1,2}$ For an $n$-component liquid mixture, $n(n+1) / 2$ different pair distribution functons $g_{v \mu}(r)$ are needed for the same goal. These are obtained from the generalized Ornstein-Zernike (OZ) equations

$$
h_{v \mu}(r)=c_{\nu \mu}(r)+\sum_{\gamma=1}^{n} \rho_{\gamma} \int d \mathbf{r}^{\prime} c_{v \gamma}\left(\left|\mathbf{r}-\mathbf{r}^{\prime}\right|\right) h_{\gamma \mu}\left(r^{\prime}\right)
$$

which introduce the corresponding direct correlation functions (DCF) $c_{v \mu}(r)$; here, $h_{v \mu}=g_{v \mu}-1$ and $\rho_{\gamma}=\rho x_{\gamma}$, with $\rho$ and $x_{\gamma}$ the total number density and partial molar fraction of species $\gamma$, respectively. A second, closure relation is then needed to complete the specification of these functions. This was obtained some time $\mathrm{ago}^{3}$ by a diagrammatical analysis of the density expansions of the PDF and DCF, which yields the exact, formal relations

$$
g_{v \mu}(r)=\exp \left[-\beta \phi_{v \mu}(r)+s_{v \mu}(r)+b_{v \mu}(r)\right],
$$

where $s_{v \mu}(r)=h_{v \mu}(r)-c_{v \mu}(r)$ and $\beta=1 / k_{B} T$. While this analysis produces a definition of the so-called "bridge" functions $b_{v \mu}(r)$ in terms of a density expansion of highly connected bridge or "elementary" diagrams, the expansion cannot be summed and the $b_{v \mu}(r)$ remain essentially unknown. This has given rise to a number of approximate closures, usually based on the summation of a subset of diagrams of the $c_{v \mu}(r)$, such as the Percus-Yevick ${ }^{4}(\mathrm{PY})$ and hypernetted-chain ${ }^{3}$ (HNC) approximations. Despite omitting entire subclasses of diagrams [e.g., $b_{v \mu}(r)=0$ for HNC], these closures produce reasonable results at low to medium densities.' They share, however, internal thermodynamic inconsistencies, such as different equations of state obtained from the virial and compressibility routes. In the past, efforts to avoid these limitations have often been based on perturbation theories, ${ }^{2}$ which recognize that in the high density and temperature region of the fluid phase diagram the fluid microscopic structure is dominated by packing effects determined by the short-range repulsive part of the interaction potential. The hard sphere fluid being the simplest model for such effects, it is usually chosen as the reference system in such perturbation theories for both simple fluids and, with additive diameters, for fluid mixtures; in addition, its structural ${ }^{5,6}$ and thermodynamic ${ }^{7}$ properties are known through analytical expressions of reasonable accuracy. The success of these theories depends on the criteria used to select the reference system parameters and is generally limited to short-range repulsive potentials and conditions of high density and temperature. ${ }^{2}$

The development in recent years of efficient algorithms ${ }^{8-10}$ for the solution of $\mathrm{OZ}$-based integral equations has encouraged new efforts in both the application of and improvements in this approach for simple liquids and their mixtures. Rogers and Young ${ }^{11}$ used the old idea of a "universal" closure (independent of the pair potential characteristics) constructed from a combination of the PY and HNC approximations such as to assure pressure consistency in the computed results. This approximation has been applied to mixtures of repulsive Lennard-Jones atoms ${ }^{12}$; a "soft core" mean spherical approximation-HNC variant of the same approach has been used to study Lennard-Jones mixtures. ${ }^{13}$ The parameter used to combine the two closures depends on the thermodynamic state being studied. In another approach, leading to the optimized reference-hypernetted chain (RHNC) equation, ${ }^{14-17}$ the bridge function $b(r)$ is modeled by the known $b_{0}(r)$ of a short-range potential $\phi_{0}(r)$. As with the perturbation theories, the analytic parametrization of the various correlation functions of the hard sphere fluid ${ }^{18-20}$ yields a convenient representation of $b_{0}(r)$ and makes this a useful reference system. The disposable 
reference system parameters are selected by minimizing the Helmholtz free energy, which leads to an important increase in the internal thermodynamic consistency of the theory. This approximation yields excellent results for simple liquids with quite diverse potentials. ${ }^{17,21}$ Recently, the hypothesis of "universality" of the bridge function has been questioned; at large separations, $b(r)$ should go as $-[g(r)-1]^{2} / 2$, which gives rise to a dependence on the particular potential being studied. ${ }^{22}$ Furthermore, the hypothesis appears to have no foundation in one-dimensional systems. ${ }^{23}$

In this work, we apply the optimized RHNC method to binary fluid mixtures described by a repulsive LennardJones (RLJ) potential and the full Lennard-Jones (LJ) potential. In Sec. II, we develop an expression for the Helmholtz free energy of mixtures as a functional of the pair correlation functions. An extremal condition on this functional yields conditions for the selection of the disposable parameters of the reference system, which is here chosen to be a mixture of additive hard spheres. The bridge functions for the hard sphere mixture are obtained in Sec. III using a parametrization of the correlation functions of this model ${ }^{5,6}$ Comments on the numerical algorithm used to solve the coupled integral equations are presented in Sec. IV, with emphasis on the modification of Gillan's ${ }^{9}$ method that we have used. Finally, we present in Sec. V the computed results obtained for the liquid mixtures noted above. They are in excellent agreement with simulation results for both structure and thermodynamics.

\section{THEORY}

The Helmholtz free energy of a fluid mixture interacting through pair potentials $\phi_{v \mu}(r)$ can be obtained by an extension of Lado's ${ }^{14,16}$ derivation for pure fluids, based on a knowledge of the structural and thermodynamical properties of a reference fluid with potentials $\phi_{\nu \mu}^{0}(r)$. To this end, it is convenient to describe the transition from reference system to real system by parametrizing the potentials in the form

$$
\begin{aligned}
& \phi_{v \mu}(r, \lambda)=\phi_{v \mu}^{0}(r)+\lambda \Delta \phi_{v \mu}(r), \\
& \Delta \phi_{v \mu}(r)=\phi_{v \mu}(r)-\phi_{v \mu}^{0}(r) .
\end{aligned}
$$

The difference in excess free energy $A$ between the real system with potentials $\phi_{v \mu}(r, 1)$ and the reference system with $\phi_{v \mu}(r, 0)$ is then given by

$$
A-A^{0}=\left(A_{1}-A_{1}^{0}\right)+\left(A_{2}-A_{2}^{0}\right)+\Delta A_{3},
$$

where

$$
\begin{aligned}
\beta A_{1} / N= & -\frac{1}{2} \rho \sum_{v \mu} x_{v} x_{\mu} \int d \mathbf{r}\left[\frac{1}{2} h_{v \mu}^{2}(r)+h_{v \mu}(r)\right. \\
& \left.-g_{v \mu}(r) \ln y_{v \mu}(r)\right] \\
\beta A_{2} / N= & -(2 \rho)^{-1} \int d \mathbf{k} /(2 \pi)^{3} \\
& \times[\ln \operatorname{Det} \widetilde{\mathbf{H}}(k)-\operatorname{Tr} \widetilde{\mathbf{H}}(k)] .
\end{aligned}
$$

In these expressions, $y_{v \mu}(r)=g_{v \mu}(r) \exp \left[\beta \phi_{v \mu}(r)\right]$, and Det and $\operatorname{Tr}$ denote the matrix determinant and trace operations, respectively, while the elements of the matrix $\mathbf{H}(k)$ are proportional to the Fourier transforms of the total correlation functions $h_{v \mu}(r)$,

$$
\begin{aligned}
& \widetilde{H}_{v \mu}(k)=\left(\rho_{\nu} \rho_{\mu}\right)^{1 / 2} \tilde{h}_{v \mu}(k), \\
& \tilde{h}_{\nu \mu}(k)=\int d \mathbf{r} h_{v \mu}(r) \exp (i \mathbf{k} \cdot \mathbf{r}) .
\end{aligned}
$$

The reference system quantities $A_{1}$ and $A_{2}$ are defined as in Eqs. (6) and (7) with the corresponding reference correlation functions. For $\Delta A_{3}$, we obtain the exact expression

$$
\begin{aligned}
\beta \Delta A_{3} / N= & -\frac{1}{2} \rho \sum_{\nu \mu} x_{\nu} x_{\mu} \int d \mathbf{r} \int_{0}^{1} d \lambda b_{\nu \mu}(r, \lambda) \\
& \times \frac{\partial g_{\nu \mu}(r, \lambda)}{\partial \lambda}
\end{aligned}
$$

in terms of the "charging" parameter $\lambda$. The RHNC approximation $^{14,15,24}$ is based on the assumption that the bridge functions are largely insensitive to the change in potential in going from $\lambda=0$ to $\lambda=1$, so that Eq. (10) can be approximately evaluated as

$$
\begin{aligned}
\beta \Delta A_{3} / N \simeq & -\frac{1}{2} \rho \sum_{\nu \mu} x_{\nu} x_{\mu} \int d \mathbf{r} b_{\nu \mu}^{0}(r) \\
& \times\left[g_{\nu \mu}(r)-g_{v \mu}^{0}(r)\right] .
\end{aligned}
$$

Equation (5) thus makes $A$ a functional of the $g_{v \mu}(r)$ and $b_{v \mu}^{0}(r)$. A variation of these then produces the change

$$
\begin{aligned}
\delta \beta A / N= & -\frac{1}{2} \rho \sum_{\nu \mu} x_{v} x_{\mu} \int d \mathrm{r}\left[h_{\nu \mu}(r)\right. \\
& \left.-\ln y_{v \mu}(r)+b_{\nu \mu}^{0}(r)-c_{v \mu}(r)\right] \delta g_{v \mu}(r) \\
& +\frac{1}{2} \rho \sum_{\nu \mu} x_{\nu} x_{\mu} \int d \mathbf{r}\left[g_{v \mu}(r)\right. \\
& \left.-g_{v \mu}^{0}(r)\right] \delta b_{\nu \mu}^{0}(r) .
\end{aligned}
$$

This variation becomes extremal if the PDF satisfy the closure (2) with the reference system bridge functions replacing the exact $b_{v \mu}(r)$, which constitutes the RHNC approximation, and if in addition the condition

$$
\sum_{\nu \mu} x_{\nu} x_{\mu} \int d \mathbf{r}\left[g_{v \mu}(r)-g_{\nu \mu}^{0}(r)\right] \delta b_{\nu \mu}^{0}(r)=0
$$

is also satisfied. Variations in the bridge functions $b_{\nu \mu}^{0}(r)$ follow from variations in the set of reference system distance and energy parameters $\{d, \epsilon\}$, so that

$$
\delta b_{v \mu}^{0}(r)=\sum_{k}\left(\frac{\partial b_{v \mu}^{0}}{\partial d_{k}}\right) \delta d_{k}+\sum_{l}\left(\frac{\partial b_{v \mu}^{0}}{\partial \epsilon_{l}}\right) \delta \epsilon_{l} .
$$

Using Eq. (14) in Eq. (13) then leads to the conditions

$$
\begin{gathered}
I_{k}=\sum_{\nu \mu} x_{\nu} x_{\mu} \int d \mathbf{r}\left[g_{\nu \mu}(r)-g_{\nu \mu}^{0}(r)\right] \frac{\partial b_{\nu \mu}^{0}(r)}{\partial d_{k}}=0 \\
J_{l}=\sum_{\nu \mu} x_{\nu} x_{\mu} \int d \mathbf{r}\left[g_{\nu \mu}(r)-g_{\nu \mu}^{0}(r)\right] \frac{\partial b_{\nu \mu}^{0}(r)}{\partial \epsilon_{l}}=0
\end{gathered}
$$

for arbitrary variations of the $\{d, \epsilon\}$; these conditions then serve as constraints that determine the values of these parameters to be used in a given calculation. It turns out 
further that Eqs. (15) lead to a notable increase in the internal thermodynamic consistency of the RHNC method. ${ }^{16} \mathrm{We}$ find by direct differentiation that

$$
\begin{aligned}
\beta p / \rho-1= & \rho \frac{\partial(\beta A / N)}{\partial \rho} \\
= & -\frac{1}{6} \rho \sum_{v \mu} x_{v} x_{\mu} \\
& \times \int d \mathrm{r} g_{v \mu}(r) r \beta \phi_{v \mu}^{\prime}(r)-\frac{1}{6} \rho \sum_{k} I_{k}
\end{aligned}
$$

and similarly

$$
\begin{aligned}
\beta U / N & =\frac{\beta \partial(\beta A / N)}{\partial \beta} \\
& =\frac{1}{2} \rho \sum_{\nu \mu} x_{\nu} x_{\mu} \int d \mathrm{r} g_{\nu \mu}(r) \beta \phi_{v \mu}(r)-\frac{1}{2} \rho \sum_{l} J_{l},
\end{aligned}
$$

which reduce to the correct forms when conditions (15) are imposed. These constraints though do not guarantee pressure consistency between the virial equation of state written above and that obtained from the compressibility,

$$
\left(\rho k_{B} T \chi_{T}\right)^{-1}=\frac{\beta \partial p}{\partial \rho}=1-\rho \sum_{\nu \mu} x_{\nu} x_{\mu} \int d \mathbf{r} c_{\nu \mu}(r)
$$

In practice, however, this inconsistency is greatly lessened compared to the PY and HNC approximations. ${ }^{17}$

We note that for hard core reference systems, which lack an energy scale, conditions Eq. (15b) are trivially satisfied.

\section{CORRELATION FUNCTIONS OF ADDITIVE HARD SPHERE MIXTURES}

Verlet and $\mathrm{Weis}^{18}(\mathrm{VW})$ formulated a parametrization of the pure hard sphere PDF based on a correction of the PY result that incorporates the Carnahan-Starling ${ }^{25}$ empirical equation of state. The analytic solution of the PY equation for additive hard sphere mixtures ${ }^{26}$ and the empirical mixtures equation of state proposed by Mansoori et al. ${ }^{7}$ (MCSL) permit an extension of the VW parametrization for such systems. ${ }^{5,6}$ One defines first the packing fraction

$$
\eta=\frac{\pi}{6} \sum_{v} \rho_{v} d_{v v}^{3}
$$

for hard spheres of diameter $d_{v v}$ and the effective sphere diameters $d_{v v}^{*}=d_{v v}(1-\eta / 16)^{1 / 3}$, which bring the oscillations of the $g_{v \mu}\left(r,\left\{d^{*}\right\}, \eta^{*}\right)$ into coincidence with simulation results. The diminished values of the resulting $g_{\nu \mu}$ near contact are then corrected with a short-range function, so that

$$
g_{v \mu}(r)=g_{v \mu}^{\mathrm{PY}}\left(r,\left\{d^{*}\right\}, \eta^{*}\right)+\Delta g_{v \mu}(r), r \geqslant d_{v \mu},
$$

where

$$
\begin{aligned}
\Delta g_{\nu \mu}(r)= & \left(A_{\nu \mu} / r\right) \exp \left[-m_{\nu \mu}\left(r-d_{\nu \mu}\right)\right] \\
& \times \cos \left[m_{\nu \mu}\left(r-d_{v \mu}\right)\right], \quad r \geqslant d_{\nu \mu} .
\end{aligned}
$$

The parameters $A_{v \mu}$ are chosen to reproduce the contact values generated by a combination of the PY result and that of scaled particle theory ${ }^{27}$ (SPT),

$$
g_{v \mu}\left(d_{v \mu}\right)=\frac{1}{3} g_{v \mu}^{\mathrm{PY}}\left(d_{\nu \mu}\right)+\frac{2}{3} g_{v \mu}^{\mathrm{SPT}}\left(d_{v \mu}\right),
$$

which assures that the virial equation of state reproduces the MCSL form. The parameters $m_{v \mu}$ are determined from the formula

$$
m_{\nu \mu} d_{v \mu}^{2}=K A_{v \mu} /\left[\eta^{*} g_{v \mu}^{\mathrm{PY}}\left(d_{\nu \mu},\left\{d^{*}\right\}, \eta^{*}\right)\right] .
$$

With $K=24$ we recover the parametrization proposed by Lee and Levesque ${ }^{5}(\mathrm{LL})$, which yields reasonable agreement between the structure factors of the true hard sphere mixture and the PY result. In the present calculations, we have obtained $K$ numerically by requiring that the computed isothermal compressibility agrees with the MCSL value,

$$
\rho k_{B} T \chi_{T}=\left(\rho k_{B} T \chi_{T}\right)_{\mathrm{MCSL}} \text {. }
$$

This ensures that the extension to mixtures of the VW parametrization is thermodynamically consistent.

In Fig. 1 we show the pair distribution functions for a binary hard sphere mixture with $d_{11} / d_{22}=0.3$ at $\eta=0.49$, as obtained from the PY equation, the present $\mathrm{VW}$ parametrization, and computer simulation. ${ }^{5}$ On the scale of this figure, our results agree with those of the $L L$ parametrization; differences between the two versions only appear in the $\tilde{c}_{v \mu}(k)$ as $k$ goes to zero, a consequence of the different compressibility choices. With the fitted $g_{v \mu}(r)$ we can compute the corresponding $s_{v \mu}(r)$ through the $\mathrm{OZ}$ equation.

Henderson and Grundke ${ }^{6}$ (HG) have fitted the func-

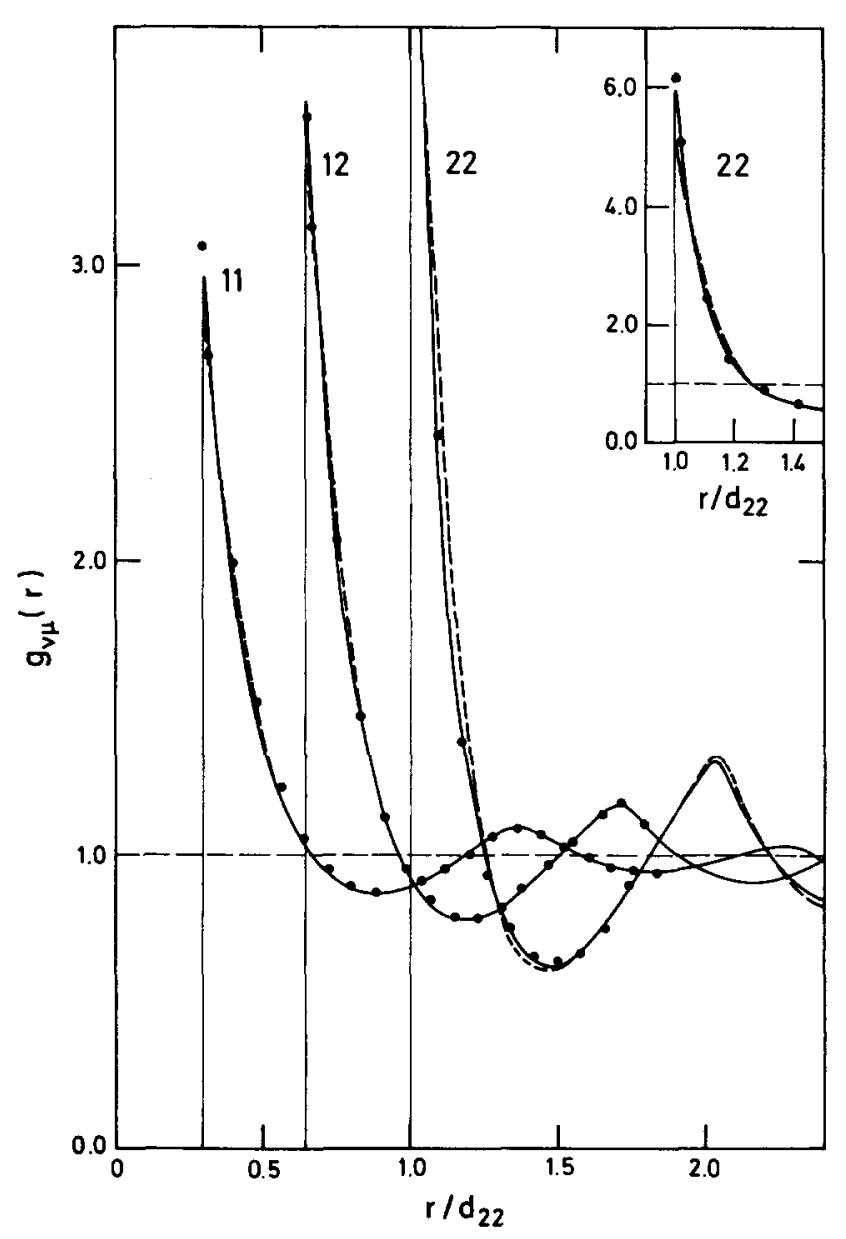

FIG. 1. Pair distribution function of an equimolar additive hard sphere mixture with $d_{11} / d_{22}=0.3$ and $\eta=0.49: \cdots \mathrm{MC}$ (Ref. 5), - VW, and - -PY. 
tions $\ln \left[y_{v \mu}(r)\right]$ in the overlap region with a third order polynomial,

$$
\ln \left[y_{\nu \mu}(r)\right]=\sum_{k=0}^{3} \alpha_{v \mu}^{(k)} r^{k}, \quad r \leqslant d_{\nu \mu} .
$$

To determine the coefficients, they generalized for mixtures some theorems and thermodynamic relations which the $y_{\nu \mu}(r)$ must satisfy:

$$
\begin{aligned}
& \ln \left[y_{v \mu}\left(r<a_{v \mu}\right)\right]=\beta \mu_{v}, \quad d_{v v}<d_{\mu \mu}, \\
& \left.\frac{\beta \partial \ln \left[y_{\nu \mu}\left(r \leqslant a_{v \mu}\right)\right]}{\partial \rho}\right|_{T}=\frac{\beta \partial p}{\partial \rho_{v}}-1, \quad d_{v v}<d_{\mu \mu},
\end{aligned}
$$

$$
d \ln \left[y_{v \mu}(r)\right] /\left.d r\right|_{r=a_{\gamma \mu}}=-\pi \delta_{v \mu} \sum_{\gamma=1}^{n} \rho_{\gamma} d_{\gamma \mu}^{2} y_{\gamma \mu}\left(d_{\gamma \mu}\right),
$$

$$
\begin{aligned}
& y_{v \mu}\left(d_{v \mu}\right)=g_{\nu \mu}\left(d_{\nu \mu}\right), \\
& y_{v \mu}^{\prime}\left(d_{\nu \mu}\right)=g_{v \mu}^{\prime}\left(d_{v \mu}\right) .
\end{aligned}
$$

In these equations, $a_{v \mu}=\left(d_{\mu \mu}-d_{v v}\right) / 2$ and $\mu_{v}$ is the excess chemical potential of species $v$. With this final fit, the bridge functions are determined over the entire range of $r$ through Eq. (2),

$$
b_{\nu \mu}(r)=\ln \left[y_{\nu \mu}(r)\right]-s_{\nu \mu}(r) .
$$

In Fig. 2, we show the hard spheres bridge functions obtained from the PY equation and the present VW-HG parametrization. One notes that the differences between the two results are similar to those of the pure fluid. ${ }^{15}$

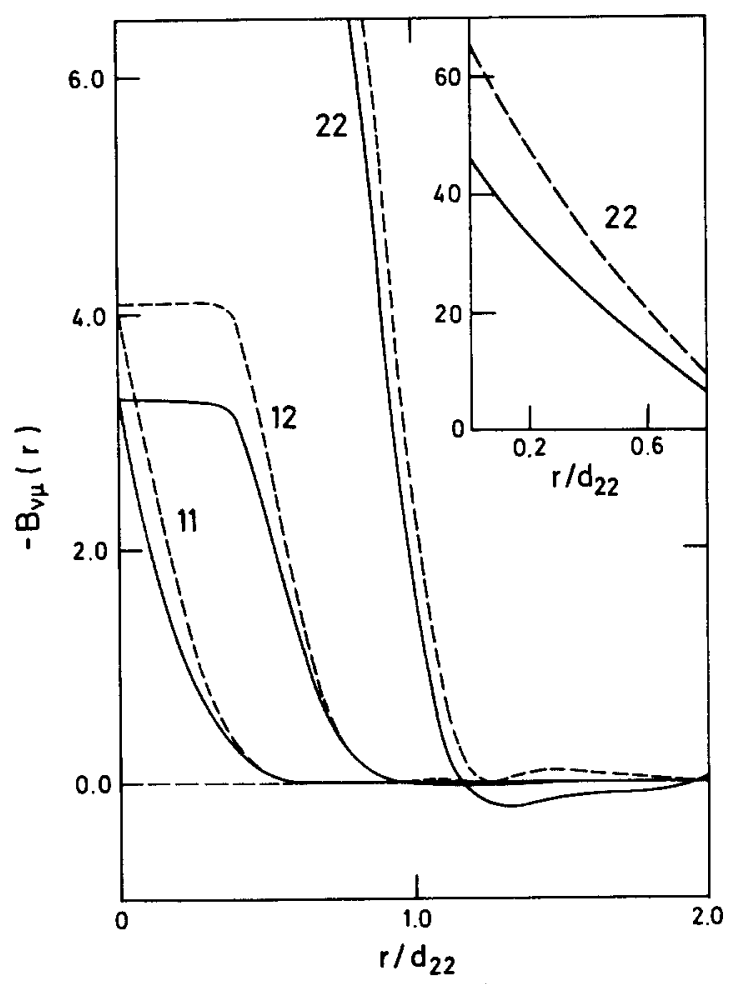

FIG. 2. Bridge functions of the hard sphere mixture of Fig. 1:-VW-HG and ... PY.

\section{CALCULATION}

The numerical solution is carried out for the functions $s_{v \mu}(r)$, with the closure relation (2) rewritten in the form

$$
\begin{aligned}
c_{v \mu}(r)= & \exp \left[-\beta \phi_{\nu \mu}(r)+s_{v \mu}(r)\right. \\
& \left.+b_{v \mu}^{o}(r)\right]-s_{v \mu}(r)-1
\end{aligned}
$$

and the $\mathrm{OZ}$ equation expressed in Fourier transform representation. In the matrix format of Eqs. (8) and (9), this reads

$$
\widetilde{\mathbf{S}}(k)=[\mathbf{I}-\widetilde{\mathbf{C}}(k)]^{-1} \widetilde{\mathbf{C}}(k)-\widetilde{\mathbf{C}}(k),
$$

where $\mathrm{I}$ is the unit matrix. The $r$ and $k$ dependent functions are evaluated on a grid of points $r_{i}=i \Delta r$ and $k_{i}=i \Delta k$, respectively, $i=1, \ldots, N$, with $\Delta r \Delta k=\pi / N$. Numerical Fourier transforms are computed in the form

$$
\begin{gathered}
\tilde{f}\left(k_{i}\right)=\left(4 \pi \Delta r / k_{i}\right) \sum_{j=1}^{N-1} r_{j} f\left(r_{j}\right) \sin \left(k_{i} r_{j}\right), \\
f\left(r_{j}\right)=\Delta k /\left(2 \pi^{2} r_{j}\right) \sum_{i=1}^{N-1} k_{i} \tilde{f}\left(k_{i}\right) \sin \left(k_{i} r_{j}\right)
\end{gathered}
$$

and are evaluated using the fast Fourier transform. ${ }^{28}$

An iteration for the solution of the $s_{v \mu}(r)$ begins with use of the current value of these functions to obtain the $c_{\nu \mu}(r)$ through Eq. (32). These are then transformed with Eq. (34a) and inserted in the $\mathrm{OZ}$ equation (33) to yield transforms of the new $s_{v \mu}(r)$, which are then inverted with Eq. (34b).

The straightforward application of this procedure is known as Picard iteration and leads to very slow convergence for the thermodynamic conditions characteristic of a dense liquid. In recent years, new algorithms have been proposed to accelerate the convergence of the iterative solution. ${ }^{8-10}$ Gillan's' method has proved to be highly effective both for its rapid convergence and insensitivity to starting conditions. It has been applied to a binary ionic mixture ${ }^{29}$ and formally generalized to multicomponent systems. ${ }^{30} \mathrm{We}$ summarize below the basis of this method and note the modifications which have been used in this work.

Gillan proposed decomposing the functions $s_{v \mu}\left(r_{i}\right)$ $=s_{v \mu i}$ into two parts, a first contribution that reflects the general form of these functions and is expressed as a linear combination of a small number of basis functions $P_{\nu \mu n}^{i}$ evaluated at $M$ points $i$ plus a second contribution for the fine corrections $\Delta s_{v \mu i}$,

$$
\begin{aligned}
& r_{i} s_{v \mu i}=\sum_{n} a_{v \mu n} P_{v \mu n}^{i}+r_{i} \Delta s_{v \mu i}, \\
& a_{v \mu n}=\sum_{i=1}^{M} Q_{v \mu n}^{i} r_{i} s_{v \mu i},
\end{aligned}
$$

where the choice of basis functions $P_{v \mu n}^{i}$ and their conjugates $Q_{v \mu n}^{i}$ used here is identical to those of Gillan. ${ }^{9}$ After a Picard or elementary cycle, we obtain a new set of coefficients $a_{v \mu n}^{\prime}$ using Eq. (36). On the basis of the difference vector $\mathbf{d}=\mathbf{a}-\mathbf{a}^{\prime}$, a new coefficient vector $\mathbf{a}^{*}$ is constructed using the classical Newton-Raphson (NR) technique, so that

$$
\mathbf{d}+\mathbf{J} \cdot\left(\mathbf{a}^{*}-\mathbf{a}\right)=0,
$$

which yields

$$
\mathbf{a}^{*}=\mathbf{a}-\mathbf{J}^{-1} \cdot \mathbf{d} \text {. }
$$


The elements of the Jacobian matrix $\mathbf{J}$ are given by

$$
\begin{aligned}
J_{v \mu n, \gamma \sigma m} & =\delta_{v \mu n, \gamma \sigma m}-\frac{\partial a_{\gamma \mu \mathrm{n}}^{\prime}}{\partial a_{\gamma \sigma m}} \\
& =\delta_{v \mu n, \gamma \sigma m}-\sum_{i j} Q_{v \mu n}^{i}\left(\frac{r_{i}}{r_{j}} \frac{\partial s_{\gamma \mu i}^{\prime}}{\partial s_{\gamma \sigma j}}\right) P_{\gamma \sigma m}^{\prime}, \\
\frac{\partial s_{v \mu i}^{\prime}}{\partial s_{\gamma \sigma j}} & =\sum_{k=1}^{N} \frac{\partial s_{\nu \mu i}^{\prime}}{\partial \tilde{s}_{v \mu k}}\left\{\sum_{(\xi \chi)} \frac{\partial \tilde{s}_{\gamma \mu k}}{\partial \tilde{c}_{\xi \chi k}} \frac{\partial \tilde{c}_{\xi \chi k}}{\partial \tilde{c}_{\gamma \sigma j}} \frac{\partial c_{\gamma \sigma j}}{\partial s_{\gamma \sigma j}}\right\},
\end{aligned}
$$

where the second sum in Eq. (40) is defined over all correlation pairs that involve atomic species $\gamma$ and $\sigma$. Calculation of the required derivatives is relatively straightforward using the equations that define a Picard cycle described above along with the matrix relation ${ }^{30}$

$$
\frac{\partial \tilde{s}_{v \mu k}}{\partial \tilde{c}_{\xi \chi^{k}}}=\left[\left(\mathbf{I}+\widetilde{\mathbf{H}}_{k}\right) \boldsymbol{\delta}^{\xi \chi}\left(\mathbf{I}+\widetilde{\mathbf{H}}_{k}\right)-\boldsymbol{\delta}^{\xi \chi}\right]_{v \mu},
$$

where the elements of the Kronecker delta matrix $\delta^{\xi x}$ are $\left(\boldsymbol{\delta}^{\xi \chi}\right)_{\eta \lambda}=\delta_{\xi \chi, \eta \lambda}$.

The NR algorithm is used iteratively after each Picard iteration until a converged set of coefficients $a_{v \mu n}$ is obtained, holding constant the fine correction, with convergence defined by the parameter

$$
\epsilon_{1}=\left[\sum_{v \mu n}\left(a_{v \mu n}-a_{v \mu n}^{\prime}\right)^{2}\right]^{1 / 2} \text {. }
$$

With convergence of the $a$ coefficients achieved, a new Picard iteration is performed to obtain a new fine correction set $\Delta s_{v \mu}(r)$ and the process is repeated until overall convergence of the $s_{v \mu}(r)$ is obtained, as measured by

$$
\epsilon_{2}=\max \left|r_{i}\left(s_{v \mu i}-s_{v \mu i}^{\prime}\right)\right| \text {. }
$$

We note that including a multiplicative $r$ factor in Eq. (35) avoids the need of computing the correlation functions at the origin, since these values are otherwise not used in the iterative solution.

Finally, the derivatives of the bridge functions with respect to the hard sphere diameters needed in Eq. (15a) have been obtained by simple finite differences,
TABLE I. Parameters of the intermolecular potentials of mixtures used in this work, in reduced units.

\begin{tabular}{lcccccc}
\hline \hline Potential & $\epsilon_{11}$ & $\epsilon_{12}$ & $\epsilon_{22}$ & $\sigma_{11}$ & $\sigma_{12}$ & $\sigma_{22}$ \\
\hline $\mathrm{RLJ}^{12}$ & 1.0000 & 1.0000 & 1.0000 & 1.0000 & 1.2000 & 1.4000 \\
$\mathrm{LJ}(\mathrm{Ar} / \mathrm{Kr})^{31}$ & 1.0000 & 1.1807 & 1.3940 & 1.0000 & 1.0335 & 1.0670 \\
$\mathrm{LJ}(\mathrm{He} / \mathrm{Xe})^{32}$ & 0.3570 & 1.0000 & 7.7800 & 0.7230 & 0.9530 & 1.1740 \\
$\mathrm{LJ}^{33}$ & 1.0000 & 1.0000 & 1.0000 & 1.0000 & 1.1250 & 1.2500 \\
\hline
\end{tabular}

$$
\begin{aligned}
\frac{\partial b_{v \mu}^{0}(r)}{\partial d_{\gamma \gamma}}= & {\left[b_{v \mu}^{0}\left(r, d_{r \gamma}+\Delta\right)\right.} \\
& \left.-b_{v \mu}^{0}\left(r, d_{\gamma \gamma}-\Delta\right)\right] /(2 \Delta) .
\end{aligned}
$$

\section{RESULTS}

The optimized RHNC equation has been solved numerically for binary mixtures of atoms interacting through the short-range $R L J$ and $L J$ potentials, with the parameters listed in Table I, for a number of thermodynamic states. The reference system for this calculation is a binary mixture of hard spheres in parametrized form, as described in Sec. III. All computations were carried out on a grid of $N=512$ points with a spacing $\Delta r=0.015$. Gillan's method of NR iterations was started from the PY solution for hard sphere mixtures and was used with the same precautions as in the pure fluid case. ${ }^{9}$ Some 15 to 20 Picard cycles were needed in each case to achieve a converged solution, defined here as $\epsilon_{1}$, $\epsilon_{2}<10^{-3}$. The constraint Eqs. (15a) defining the reference systems were satisfied to an accuracy of $10^{-2}$; greater precision falls within the numerical uncertainties of the calculation and has scarcely any effect on the final computed thermodynamic and structural properties.

\begin{tabular}{|c|c|c|c|c|c|c|c|}
\hline $\begin{array}{l}\text { Thermodynamic } \\
\text { state }\end{array}$ & Approximation & $U / N k T$ & $p V / N k T$ & $A / N k T$ & $\beta \partial p / \partial \rho$ & $d_{1}$ & $d_{2}$ \\
\hline \multirow{2}{*}{$\begin{array}{l}k T / \epsilon=0.501 \\
\Gamma=0.28 \\
x_{1}=0.5\end{array}$} & VW/GH & 0.735 & 3.94 & 1.99 & 9.24 & 1.0169 & 1.3979 \\
\hline & $R Y^{12}$ & & 3.92 & & 9.18 & & \\
\hline \multirow{2}{*}{$\begin{array}{l}k T / \epsilon=0.501 \\
\Gamma=0.482 \\
x_{1}=0.5\end{array}$} & $\mathrm{VW} / \mathrm{GH}$ & 2.330 & 10.33 & 4.92 & 32.43 & 0.9810 & 1.3428 \\
\hline & $R \mathbf{Y}^{12}$ & & 10.24 & & 32.63 & & \\
\hline \multirow{3}{*}{$\begin{array}{l}k T / \epsilon=0.501^{\mathrm{a}} \\
\Gamma=0.635 \\
x_{1}=0.5\end{array}$} & PY & 4.480 & 18.92 & 8.28 & 85.28 & 0.9560 & 1.3070 \\
\hline & $\mathrm{VW} / \mathrm{GH}$ & 4.780 & 20.14 & 8.78 & 73.41 & 0.9561 & 1.3010 \\
\hline & $\mathbf{R} \mathbf{Y}^{12}$ & & 20.02 & & 73.48 & & \\
\hline $\begin{array}{l}k T / \epsilon=0.423 \\
\Gamma=0.8635 \\
x_{1}=0.75\end{array}$ & VW/GH & 5.365 & 22.46 & 9.71 & 85.56 & 0.9446 & 1.2888 \\
\hline
\end{tabular}

The final results do depend on whether the PY hard spheres solution or the parametrized VW-HG fit is used for the reference system. This is shown in Table II for the equimolar RLJ mixture with $\Gamma=\rho \sigma_{11}^{3}(\beta \epsilon)^{1 / 4}=0.635$ and $k_{B} T / \epsilon=0.501$. The underestimation of $g_{v \mu}^{0}(r)$ near contact produced by the PY equation leads to larger effective

TABLE II. Computed thermodynamic properties of RLJ mixtures.

aC value $p V / N k T=20.22$ (Ref. 12). 
TABLE III. Dependence of the thermodynamic properties of the RLJ model on the reference system diameter $d_{22}$ for the state with $x_{1}=0.5$, $\Gamma=0.635, k_{B} T / \epsilon=0.501$, and $d_{11}=0.95605$.

\begin{tabular}{lllllll}
\hline \hline$d_{22}$ & $I_{1}$ & $I_{2}$ & $p V / N k T A / N k T$ & $\beta \partial p / \partial \rho$ & $U / N k T$ \\
\hline 1.3145 & -0.49 & -1.86 & 19.83 & 8.650 & 77.35 & 4.700 \\
1.3010 & $10^{-4}$ & $-10^{-3}$ & 20.14 & 8.720 & 73.41 & 4.780 \\
1.2876 & +0.43 & +1.53 & 20.40 & 8.670 & 69.87 & 4.850 \\
\hline
\end{tabular}

TABLE IV. Thermodynamic consistency of the RHNC approximation for the system of Table III.

\begin{tabular}{cccc}
\hline \hline$\Gamma$ & $p V / N k T$ & $\beta \partial p / \partial \rho$ & $B_{v} / B_{c}$ \\
\hline 0.637 & 20.30 & 74.16 & \\
0.635 & 20.14 & 73.41 & 0.99 \\
0.633 & 19.97 & 72.67 & \\
\hline
\end{tabular}

rABLE V. Computed thermodynamic properties of equimolar LJ mixtures: First row, $\mathrm{Ar}-\mathrm{Kr}$ at $T=115.8 \mathrm{~K}$ (Ref. 31); Second row, He-Xe near thermodynamic conditions of phase separation (Ref. 32).

\begin{tabular}{|c|c|c|c|c|c|c|c|c|c|}
\hline \multicolumn{2}{|c|}{$\begin{array}{l}V\left(\mathrm{~cm}^{3} \mathrm{~mol}^{-1}\right) \\
\text { Theory } \mathrm{MC}\end{array}$} & \multicolumn{2}{|c|}{$\begin{array}{c}U / N k T \\
\text { Theory MC }\end{array}$} & \multicolumn{2}{|c|}{$\begin{array}{l}p V / N k T \\
\text { Theory MC }\end{array}$} & \multirow{2}{*}{$\begin{array}{l}A / N k T \\
-3.8981 \\
-0.0714\end{array}$} & \multirow{2}{*}{$\begin{array}{r}\beta \partial p / \partial \rho \\
14.58 \\
2.37\end{array}$} & \multirow{2}{*}{$\begin{array}{c}d_{1} / \sigma \\
1.0121 \\
0.6157\end{array}$} & \multirow{2}{*}{$\begin{array}{l}d_{2} / \sigma \\
1.0881 \\
1.1198\end{array}$} \\
\hline 33.09 & 32.82 & $\begin{array}{l}-7.014 \\
-1.018\end{array}$ & -7.101 & $\begin{array}{l}0.010 \\
1.254\end{array}$ & $\begin{array}{l}0.003 \\
1.234\end{array}$ & & & & \\
\hline
\end{tabular}

TABLE VI. Composition dependence of the thermodynamic properties of $\mathrm{LJ}$ mixtures for $p \sigma_{11}^{3} / \epsilon_{11}=0.5$ and $k_{B} T / \epsilon_{11}=1.0$ (Ref. 33).

\begin{tabular}{cccccccccc}
\hline \multicolumn{10}{c}{$\rho \sigma_{11}^{3}$} \\
$x_{1}$ & Theory & MD & Theory & MD & $A / N k T$ & $p V / N k T$ & $\beta \partial p / \partial \rho$ & $d_{11} / \sigma_{11}$ & $d_{22} / \sigma_{11}$ \\
\hline 0.75 & 0.6248 & 0.6256 & -5.301 & -5.314 & -2.552 & 0.803 & 12.12 & 0.9986 & 1.2732 \\
0.50 & 0.5303 & 0.5302 & -5.347 & -5.356 & -2.547 & 0.941 & 13.06 & 0.9963 & 1.2648 \\
0.25 & 0.4602 & 0.4595 & -5.401 & -5.409 & -2.546 & 1.087 & 13.87 & 0.9860 & 1.2620 \\
\hline
\end{tabular}

packings (i.e., $d_{22}^{\mathrm{PY}}>d_{22}^{\mathrm{VW}}$ ), which is reflected in the larger magnitude of $b_{\nu \mu}^{\text {PY }}(r)$ seen in Fig. 2 and affects the final properties of the system of interest.

Additional results for other thermodynamic states of the RLJ mixture are given in Table II, along with similar data from the recent work of Hansen and Zerah ${ }^{12}$ using the RY ${ }^{11}$ closure. The present calculation yields a slightly larger compressibility factor $p V / N k_{B} T$; both calculations are in good agreement with the single datum available from Monte Carlo simulation. ${ }^{12}$

In Table III we show the variation of the thermodynamic properties of the abovementioned RLJ mixture with changes in the diameter $d_{22}$ of the second component of the reference system mixture. It can be seen that as the integrals $I_{k}$ of Eq. (15a) pass through zero the free energy is made extremal, though here it becomes maximal rather than minimal.

Table IV presents a test of pressure consistency in the computed results for the same state discussed above. Here the bulk modulus $B=V(\partial p / \partial V)_{T}$ obtained directly from Eq. (18) is compared with the same quality computed by a finite-difference derivative of the virial pressure. The good agreement found between the two values in this case may be fortuitous; a systematic check of this consistency was not made.
Table V compares the computed thermodynamics for LJ models of real mixtures with recent simulation results using these potentials. Differences between the present values and the Monte Carlo ( $N p T$ ensemble) data of $\mathrm{Mc}$ Donald ${ }^{31}$ for the Ar-Kr mixture are larger than for the other cases studied. (The computed solution was here obtained by varying the input density until the resulting computed pressure reasonably matched the $N p T$ simulation value.) The $\mathrm{He}-\mathrm{Xe}$ mixture has been studied by Fiorese ${ }^{32}$ for a thermodynamic state near phase separation, with $k_{B} T / \epsilon=10.685$, $\rho \sigma^{3}=0.49224$, and $x_{\mathrm{He}}=0.5053$. The theoretical compressibility factor is in good agreement with the Monte Carlo (NVT ensemble) result, ${ }^{32}$ as are the pair distribution functions, shown in Fig. 3. Small differences are seen in this figure in the first minimum of the cross correlation function and beyond the minimum in the distribution function of the larger component.

In Table VI we show the effects of variations in composition $\left(x_{1}=0.75,0.5,0.25\right)$ on the thermodynamic properties of the LJ mixture recently studied extensively by Gupta ${ }^{33}$ using molecular dynamics ( $N p T$ ensemble) simulation. Differences between the computed densities and internal energies and the simulation data are here within $0.2 \%$. The good agreement with simulation in this calculation is also seen in the distribution function shown in Fig. 4. 


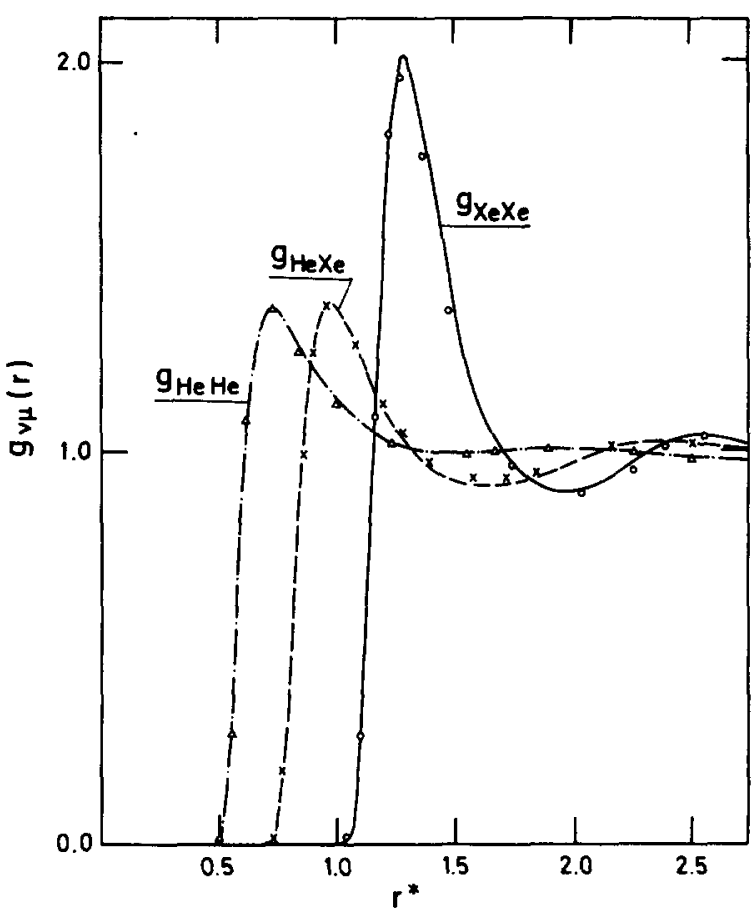

FIG. 3. Computed pair distribution functions of the $\mathrm{L}$ mixture for the HeXe model. Discrete points are simulation results (Ref. 32); $r^{*}=r /$ $\left(x_{1} \sigma_{1}^{3}+x_{2} \sigma_{2}^{3}\right)^{1 / 3}$.

\section{CONCLUSIONS}

We have extended and applied the optimized RHNC approximation for the structural and thermodynamic properties of binary mixtures whose atoms interact through spherically symmetric short-range potentials of RLJ and $\mathrm{LJ}$ type. The reference system employed for the calculation is a binary mixture of additive hard spheres whose thermodynamic properties are fixed by the MCSL equation of state, ${ }^{7}$ with the reference bridge functions obtained via the parametrizations of Lee and Levesque ${ }^{5}$ and Henderson and Grundke, ${ }^{6}$ modified to produce thermodynamic consistency of the reference model. The computed results that are obtained are in excellent agreement with available simulation data $^{31-33}$ and in the case of the RLJ potential also with the earlier calculation of Hansen and Zerah ${ }^{12}$ based on the Rogers-Young ${ }^{11}$ closure. They are however very sensitive to the precise knowledge of the reference system properties.

\section{ACKNOWLEDGMENTS}

We are grateful to S. Gupta and J. Haile for making the results of their simulation calculations available to us prior to publication. This work was supported by the U. S.-Spain Joint Committee for Scientific and Technological Cooperation under Grant No. CCB-84-09015, the Ministerio de Educación y Ciencia under Grant No. 1860/82 (CAYCYT), and the National Science Foundation under Grant No. CHE-84-02144. We thank the Computing Center of the Universidad Complutense for generous access to its computing facilities.

'J. P. Hansen and I. R. McDonald, Theory of Simple Liquids (Academic, New York, 1976).

${ }^{2}$ J. A. Barker and D. Henderson, Rev. Mod. Phys. 48, 587 (1976).

${ }^{3}$ J. M. J. VanLeeuwen, J. Groeneveld, and J. de Boer, Physica (Utrecht) 25, 792 (1959); T. Morita and K. Hiroike, Prog. Theor. Phys. 23, 1003 (1960); M. S. Green, J. Chem. Phys. 33, 1403 ( 1960); E. Meeron, J. Math. Phys. 1, 192 (1960).

${ }^{4}$ J. K. Percus and G. L. Yevick, Phys. Rev. 110, 1 (1958); G. Stell, in The Equilibrium Theory of Classical Fluids, edited by H. L. Frisch and J. L. Lebowitz (Benjamin, New York, 1964).

${ }^{5}$ L. L. Lee and D. Levesque, Mol. Phys. 26, 1351 (1973).

${ }^{6}$ E. W. Grundke and D. Henderson, Mol. Phys. 24, 269 (1972).

${ }^{7}$ G. A. Mansoori, N. F. Carnahan, K. E. Starling, and T. W. Leland, Jr., J. Chem. Phys. 54, 1523 (1971).

${ }^{8} \mathrm{~K}$. Ng, J. Chem. Phys. 61, 2680 (1974).

${ }^{9}$ M. J. Gillan, Mol. Phys. 38, 1781 (1979).

${ }^{10}$ S. Labik, A. Malijevsky, and P. Vonka, Mol. Phys. 56, 709 (1985).

${ }^{11}$ F. J. Rogers and D. A. Young, Phys. Rev. A 30, 999 (1984).

${ }^{12}$ J. P. Hansen and G. Zerah, Phys. Lett. A 108, 277 (1985).

${ }^{13}$ G. Zerah and J. P. Hansen, J. Chem. Phys. 84, 2336 (1986).

${ }^{14}$ F. Lado, Phys. Rev. A 8, 2548 (1973).

${ }^{15}$ Y. Rosenfeld and N. W. Ashcroft, Phys. Rev. A 20, 1208 (1979).

${ }^{16}$ F. Lado, Phys. Lett. A 89, 196 (1982).

${ }^{17}$ F. Lado, S. M. Foiles, and N. W. Ashcroft, Phys. Rev. A 28, 2374 (1983).

${ }^{18}$ L. Verlet and J. J. Weis, Phys. Rev. A 5, 939 (1972).

${ }^{19}$ D. Henderson and E. W. Grundke, J. Chem. Phys. 63, 601 (1975).

${ }^{20}$ E. Waisman, Mol. Phys. 25, 45 (1973).

${ }^{21}$ J. Talbot, J. L. Lebowitz, E. M. Waisman, D. Levesque, and J. J. Weis, J. Chem. Phys. 85, 2187 (1986).

${ }^{22}$ S. M. Foiles, N. W. Ashcroft, and L. Reatto, J. Chem. Phys. 80, 4441 (1984).

${ }^{23}$ M. Robert and R. Viswanathan, J. Chem. Phys. 85, 6068 (1986).

${ }^{24}$ F. Lado, J. Chem. Phys. 59, 4830 (1973). 
${ }^{25}$ N. F. Carnahan and K. E. Starling, J. Chem. Phys. 51, 635 (1969).

${ }^{26}$ J. L. Lebowitz, Phys. Rev. A 133, 895 (1964); K. Hiroike, J. Phys. Soc. Jpn. 27, 1415 (1970).

${ }^{27}$ J. L. Lebowitz, E. Helfand, and E. Praestgaard, J. Chem. Phys. 43, 774 (1965).

${ }^{28}$ E. O. Brigham, The Fast Fourier Transform (Prentice-Hall, Englewood Cliffs, 1974), Chap. 10.

${ }^{29}$ G. M. Abernethy and M. Gillan, Mol. Phys. 39, 839 (1980).
${ }^{30}$ E. Enciso, Mol. Phys. 56, 129 (1985).

${ }^{31}$ I. R. McDonald, Mol. Phys. 23, 41 (1972).

${ }^{32}$ G. Fiorese, Thèse de Doctorat d'Etat, Université Pierre et Marie Curie, Paris, 1981 (unpublished)

${ }^{33}$ S. Gupta, Ph. D. dissertation, Clemson University, 1984 (unpublished). The molecular dynamics simulations of mixtures discussed in this work were carried out with 864 particles and $14000,12000,14000$ time steps, respectively, for the molar fractions $0.75,0.5$, and 0.25 . 